\title{
Simple and Effective GNSS Spatial Processing Using a Low-Cost Compact Antenna Array
}

\author{
Ezequiel A. Marranghelli, Ramón López La Valle, and Pedro A. Roncagliolo
}

\begin{abstract}
A compact planar four-element antenna array that can be used to improve robustness and accuracy in applications of satellite-based navigation systems which require simple, lowcost, lightweight but also efficient antenna solutions is proposed. By introducing an orthonormal transformation in the digital signal processing stage, it is possible to simplify the effect of the distorted in-situ antenna radiation patterns obtaining an equivalent almost ideal phased array response. The actual prototype designed under this concept is a $14 \mathrm{~cm} \times 14 \mathrm{~cm}$ single layer microstrip antenna array for the GNSS L1/E1-bands that achieves good total antenna efficiency without employing any additional matching/decoupling stage. Promising results obtained through numerical simulations and on-field experiments showing effective direction of arrival estimations and mitigation of radio frequency interferences with simple spatial processing algorithms applied after the proposed signal transformation are presented.
\end{abstract}

Index Terms-Compact Antenna Array, GNSS, Orthonormal Transformation, Spatial Processing, In-situ Radiation Patterns.

\section{INTRODUCTION}

Global Navigation Satellite Systems (GNSS) receivers are susceptible to radio frequency interferences (either intentional or unintentional) that overlap GNSS signals in-band since their power levels are usually stronger at the receiver location due to their sources can be much closer than the satellites [1], [2]. In consequence, radio frequency (RF) interferences are a cause of a significant concern to many GNSS users. There are several techniques for interference mitigation [3]. In particular, antenna arrays (AA's) are used to reject interference in the space domain, which can be further combined with signal processing in time or frequency domains if required [4]-[8]. Currently, AA's are found in numerous GNSS robust and precise navigation applications. However, their use in many relatively small or lightweight vehicles, like modern unmanned aerial ones, is not usually possible because of the required space, power consumption and/or implementation complexity, and cost. For that reason, several new array designs addressing some of the previously mentioned issues have been recently proposed [9]-[12]. Nevertheless, small arrays with a reduced number of elements still require an accurate knowledge of the actual array response, i.e. the in-situ radiation patterns (RP's) of its elements. This information is necessary in most cases to guarantee or improve the availability, accuracy and/or integrity of the signals for GNSS receivers [13]. Then, considerably

This work was supported by the Agencia Nacional de Promoción Científica y Tecnológica under Grant PICT-2014-1232, and by the National University of La Plata under Grant I-209.

Authors are with UIDET SENyT, Departamento de Electrotecnia, Facultad de Ingeniería, Universidad Nacional de La Plata, La Plata, 1900, Argentina, e-mail: ezequiel.marranghelli@ing.unlp.edu.ar. large storage and processing capabilities must be provided at the receiver to manage the calibration data of the array [14].

In order not to depend on the accurate knowledge of the response of the individual antenna elements and to reduce the complexity of the signal processing stages of the receiver, different compensation methods have been proposed. The idea is to translate the actual AA response into approximately equally behaved elements so that it can be controlled as an ideal phased array [15]. However, the complexity reduction comes at the cost of some performance loss. There are several compensation methods available (see [16], [17] and references therein). These methods are not much concerned about the AA design nor the transformed AA user requirements. Thus, their tradeoff may involve: poor compensation quality, illconditioned transformations, and degraded statistical accuracy. The objective of this work is to propose a new array design criterium and the associated signal processing strategy to make its use practical and efficient. This approach was introduced in [18], and its key element is an orthonormal transformation that compensates the array response. It is performed multiplying the signal vector by a unitary matrix so that the statistical properties of the received noise remain unaltered, which guarantees optimal conditions for the following array signal processing. In order to achieve this objective, the actual array and the compensated -virtual- array must be jointly designed. In [18], this fact was illustrated with a two-antenna array example. While the unitary transformation can be always obtained for a given set of directions of arrival (DOA's), in general different transformations are required for different sets of DOA's. In this work, our goal is to design a four-element AA such that the associated virtual AA obtained with a single unitary transformation be useful at the main part of the upper radiation hemisphere. Moreover, to achieve a better fitting in such a large spatial region while keeping as low as possible the complexity of its model, we include a parametric virtual AA with two tuning parameters. Hence, in order to ease this cumbersome design task, a quasi-automated procedure has been developed and presented here as a novel contribution.

Following the aforementioned procedure, we have designed an actual four-antenna array and its associated transformation that allows simplified spatial processing. It is a $14 \mathrm{~cm} \times 14 \mathrm{~cm}$ single layer array for the GNSS L1/E1-bands implemented on a low-cost hydrocarbon ceramic substrate. In contrast to the approach proposed by other authors, we do not include any matching and/or decoupling stage [10], [19]-[21]. This fact considerably simplifies the design and manufacturing of the array, which in turn implies a very significant cost reduction while still achieving a good total antenna efficiency compared 
to other recent works, about $-2.7 \mathrm{~dB}$. Therefore, this prototype is compact, low-cost, lightweight and efficient. And when used jointly with the unitary transformation, it does not require a large memory nor high processing capabilities of the receiver at the same time. The AA design, the obtained virtual AA parameters, and their corresponding unitary transformation were validated by simulations and with on-field experiments. We show that if the actual array is employed for interference/jamming/spoofing/meaconing localization and mitigation with well known spatial array processing techniques, the obtained results are very satisfactory and in good agreement with simulations.

The rest of the paper is organized as follows. In Section II, a description of the proposed orthonormal transformation method and the details of its implementation are addressed. Moreover, the relationship between the characteristics of a given array design and the corresponding attainable compensated array response is explained. And it is stated that both the actual and the compensated responses are part of the design criteria. In Section III, the compact GNSS microstrip array design is proposed, its actual response is simulated, and the compensated response is obtained. In Section IV, the array design performance is tested by simulation in demanding interference rejection scenarios and compared to other stateof-the-art compensation schemes. In Section V, experimental data obtained with the actual array prototype are presented, which confirm the results of the previous section. Finally, in Section VI, the most relevant conclusions are drawn.

\section{Compens ation Scheme}

\section{A. Design Methodology}

Consider an array of $N$ identical antennas aligned with the xy-plane. Thus, the $n$-th RP for a given direction of arrival (DOA) can be calculated as [15], [22],

$$
h_{n}(\theta, \phi)=s_{n}(\theta, \phi) g_{n}(\theta, \phi),
$$

where,

- $\theta$ and $\phi$ represent elevation and azimuth coordinates respectively, where $\theta=0^{\circ}$ is $\mathrm{z}$-axis aligned,

- $g_{n}$ is the $n$-th embedded far-field gain pattern centered at its own geometric center:

$$
\because g_{n}(\theta, \phi)=\left|g_{n}(\theta, \phi)\right| \angle g_{n}(\theta, \phi),
$$

- $s_{n}$ is the $n$-th element of the steering vector which is unitary and phase dependent on the geometry of the array:

$$
\because s_{n}(\theta, \phi)=e^{j \frac{2 \pi d_{n}}{\lambda} \sin (\theta) \cos \left(\phi-\varphi_{n}\right)},
$$

being $\lambda$ the carrier wavelength of the received wave, and $\left(d_{n}, \varphi_{n}\right)$ the polar coordinates of the geometric centers of each antenna referred to the geometric center of the array.

The actual RP's $h_{n}(\theta, \phi)$ can be stacked together in a $\mathrm{N}$ dimentional column vector, labeled $\mathbf{h}(\theta, \phi)$, usually called the array manifold. Similarly, $\mathbf{s}(\theta, \phi)$ and $\mathbf{g}(\theta, \phi)$ vectors can be defined. Additionally, it is convenient to define the correlation function of the response vector for all possible pairs of DOA's, $\left(\theta^{\prime}, \phi^{\prime}\right)$ and $\left(\theta^{\prime \prime}, \phi^{\prime \prime}\right)$. That is,

$$
r\left(\theta^{\prime}, \phi^{\prime}, \theta^{\prime \prime}, \phi^{\prime \prime}\right)=\left\langle\mathbf{h}\left(\theta^{\prime}, \phi^{\prime}\right), \mathbf{h}\left(\theta^{\prime \prime}, \phi^{\prime \prime}\right)\right\rangle,
$$

where $\left\langle\mathbf{h}\left(\theta^{\prime}, \phi^{\prime}\right), \mathbf{h}\left(\theta^{\prime \prime}, \phi^{\prime \prime}\right)\right\rangle=\mathbf{h}^{H}\left(\theta^{\prime}, \phi^{\prime}\right) \mathbf{h}\left(\theta^{\prime \prime}, \phi^{\prime \prime}\right)$ is the inner product. This function maps the correlation present in the array response for all the possible combinations of any pair of DOA's. Its magnitude indicates how collinear two particular vectors are in two different DOA's. If these two orientations are highly correlated, they can be easily confused performing spatial processing. In other words, it describes the capacity of an AA to discriminate every pair of orientations in far-field.

The purpose of the compensation method is to obtain an equally behaved phased array by means of the proposed AA by design and the associated linear transformation produced by a unitary matrix $W$. That is,

$$
W \mathbf{h}(\theta, \phi)=\tilde{\mathbf{h}}(\theta, \phi) \approx \stackrel{\circ}{\mathbf{h}}(\theta, \phi),
$$

where $\tilde{\mathbf{h}}$ is the compensated array response, and $\mathbf{h}$ is the ideal -desired- objective phased array response. The matrix $W$ is constrained to be unitary so that it does not alter the noise power correlation structure of the array measurements [18]. The received noise is considered to be mainly due to the thermal agitation in the antenna and front-end stages, which can be assumed independently and identically distributed among the channels. The unitary matrix keeps these noise properties unaltered, and therefore the typical assumptions of array signal processing are still valid [2], [15], [16].

The first step to obtain $W$ is to define a model for the objective array response $\mathbf{h}$ so that the following signal processing stages can be handled conveniently. In [18], we showed that a useful transformation is feasible, i.e. possible to be obtained, if the correlation function (2) at the DOA's of interest of the actual and objective arrays are highly similar. That is,

$$
r\left(\theta^{\prime}, \phi^{\prime}, \theta^{\prime \prime}, \phi^{\prime \prime}\right) \approx \stackrel{\circ}{r}\left(\theta^{\prime}, \phi^{\prime}, \theta^{\prime \prime}, \phi^{\prime \prime}\right),
$$

where $\stackrel{r}{r}$ is the correlation function of the objective array. Therefore, $\mathbf{h}$ must be essentially designed to satisfy (4) since this leads to a straightforward way to search for the transformation matrix $W$. Since it is very possible that the proposed model does not perfectly match to any practical realization of an AA, we define $\tilde{\mathbf{h}}$ as the transformed -or virtual- array response. Notice that $\tilde{\mathbf{h}}$ has exactly the same correlation function of $\mathbf{h}$. Hence, our AA design criteria is given by its correlation function, rather than by its RP's.

Fig. 1 shows a block diagram of a generic GNSS receiver with a transformation stage for compensating the AA response at a given DOA. The required computational complexity is significantly reduced if a global transformation is performed. Hence, the matrix $W$ is defined only once and solely updated if considerable changes are evidenced at the RP's.

\section{B. Numerical Implementation}

Henceforth, the correlation function of the AA $r\left(\theta^{\prime}, \phi^{\prime}, \theta^{\prime \prime}, \phi^{\prime \prime}\right)$ is to be found numerically. To perform a global compensation of the upper hemisphere, a grid is defined in the range $0 \leq \theta<\pi / 2$ and $0 \leq \phi<2 \pi$ with step angles $\Delta_{\theta}$ and $\Delta_{\phi}$, resulting in a set $\left\{\left(\theta_{p}, \phi_{p}\right)\right\}_{p=1}^{P}$ of 


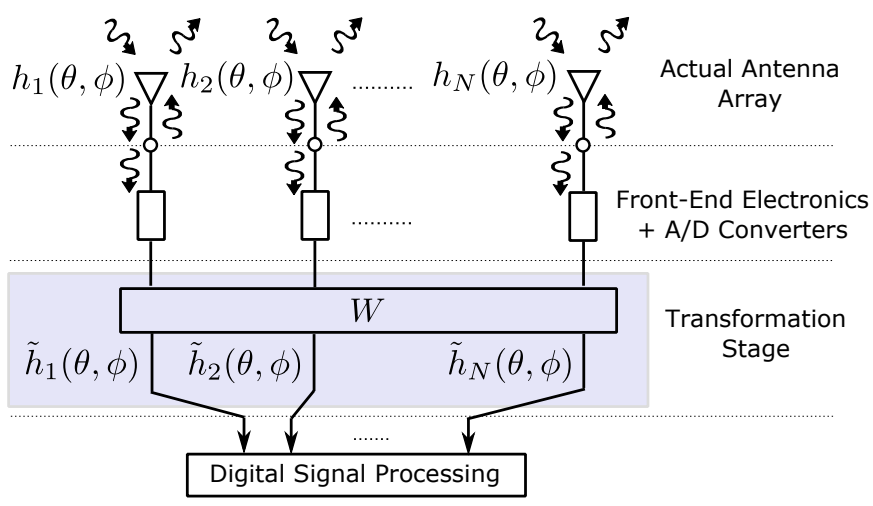

Fig. 1. GNSS receiver model with AA and compensation stage.

$P$ different DOA's for fitting purposes. The RP's $h_{n}(\theta, \phi)$ are evaluated at these DOA's of interest for the compensation and then written in matrix notation,

$H=\left[\mathbf{h}_{1}, \ldots, \mathbf{h}_{P}\right]=\left[\begin{array}{ccc}h_{1,1} & \ldots & h_{1, P} \\ : & \ldots & : \\ h_{N, 1} & \ldots & h_{N, P}\end{array}\right] ; \quad h_{n, p}=h_{n}\left(\theta_{p}, \phi_{p}\right)$,

as well as the gain $G$ and steering $S$ matrices,

$$
\begin{gathered}
S=\left[\mathbf{s}_{1}, \ldots, \mathbf{s}_{P}\right]=\left[\begin{array}{ccc}
s_{1,1} & \ldots & s_{1, P} \\
: & \ldots & : \\
s_{N, 1} & \ldots & s_{N, P}
\end{array}\right] ; \quad s_{n, p}=s_{n}\left(\theta_{p}, \phi_{p}\right), \\
G=\left[\mathbf{g}_{1}, \ldots, \mathbf{g}_{P}\right]=\left[\begin{array}{ccc}
g_{1,1} & \ldots & g_{1, P} \\
: & \ldots & : \\
g_{N, 1} & \cdots & g_{N, P}
\end{array}\right] ; \quad g_{n, p}=g_{n}\left(\theta_{p}, \phi_{p}\right) .
\end{gathered}
$$

Thus, $H=S \circ G$, where $\circ$ is the Hadamard product. The particular correlation matrix of the designed AA is

$$
R=H^{H} H,
$$

which contains a total of $P \times P$ elements. The corresponding correlation matrix of the objective array response $H$ is,

$$
\stackrel{\circ}{R}=\stackrel{\circ}{H} \stackrel{\circ}{H},
$$

and the euclidian distance between both correlation matrices can be evaluated through the Frobenius norm,

$$
\gamma=\|R-\stackrel{\circ}{R}\|_{F}^{2},
$$

which represents the cost function that must be minimized by establishing a proper design of $H$, i.e. the actual AA, and a

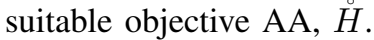

\section{COMPaCt Microstrip Antenna Array}

\section{A. Designed Array}

Typically, GNSS receivers employ microstrip antennas since they have good hemispherical radiation properties, which is important to maximize the visibility range of available satellites. In addition, they can be easily designed to achieve circular polarization, they are low cost, mechanically robust and have low profile. In particular, we selected a microstrip AA of square patches with truncated corners to obtain right hand circular polarization (RHCP) [23].
The microstrip AA was planned to be printed on a RO4350B substrate with relative dielectric permitivity of $\varepsilon_{r}=3.71$ at the center frequency of the L1/E1 band of GPS (Global Positioning System) and Galileo, $f_{c}=1.57542 \mathrm{GHz}$, [24]. The proposed array structure is very simple: four antennas in a square disposition with the same substrate and ground plane. Each antenna has a single coaxial feed. They are separated by a distance of $d=\lambda / 3$, being $\lambda$ the wavelength of $f_{c}$. The AA and its parameters of interest are shown in Fig. 2, where the final dimensions were obtained by simulation.

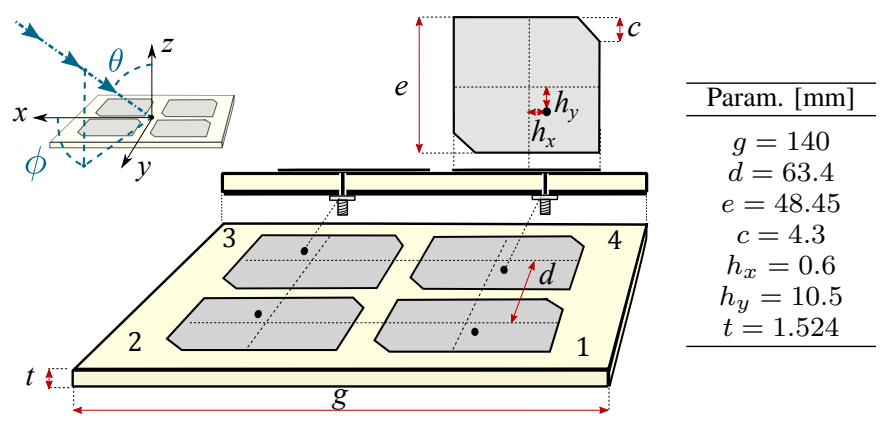

Fig. 2. Patch antenna element and four-element linear array.

The simulated AA was optimized to be matched at $f_{c}$ with a polarization bandwidth of around $10 \mathrm{MHz}$. Indeed, the obtained design shows a wider bandwidth -of about $25 \mathrm{MHz}-$ taking $\left|\mathcal{S}_{i k}\right| \lesssim-10 \mathrm{~dB}$ as the threshold for the scattering parameters, shown in Fig. 3. Given the array symmetry, the curve $\mathcal{S}_{41}$ overlaps $\mathcal{S}_{32}$, and $\mathcal{S}_{43}$ overlaps $\mathcal{S}_{21}$.

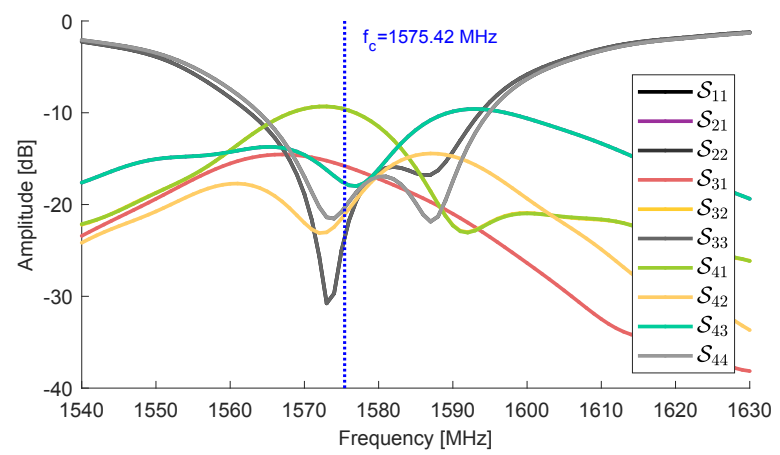

Fig. 3. Simulated scattering parameters.

The most relevant simulated parameters at the center frequency $f_{c}$ of the designed AA are presented in Table I. The simulated realized gain patterns are shown in Fig. 4 where quite different RP's can clearly be seen.

TABLE I

ANTENNA ARRAY MAIN PARAMETERS.

\begin{tabular}{|c|c|c|}
\hline Parameters \Antennas & $1 \& 3$ & $2 \& 4$ \\
\hline RHCP Realized Gain at main lobe & $4.4 \mathrm{~dB}$ & $4.5 \mathrm{~dB}$ \\
\hline RHCP main lobe elevation angle & $31^{\circ}$ & $32^{\circ}$ \\
\hline RHCP Beamwidth at main lobe & $82.4^{\circ}$ & $70^{\circ}$ \\
\hline Axial ratio at main lobe & $2.4 \mathrm{~dB}$ & $2.9 \mathrm{~dB}$ \\
\hline RHCP Total Efficiency & $-2.7 \mathrm{~dB}$ & $-2.6 \mathrm{~dB}$ \\
\hline
\end{tabular}




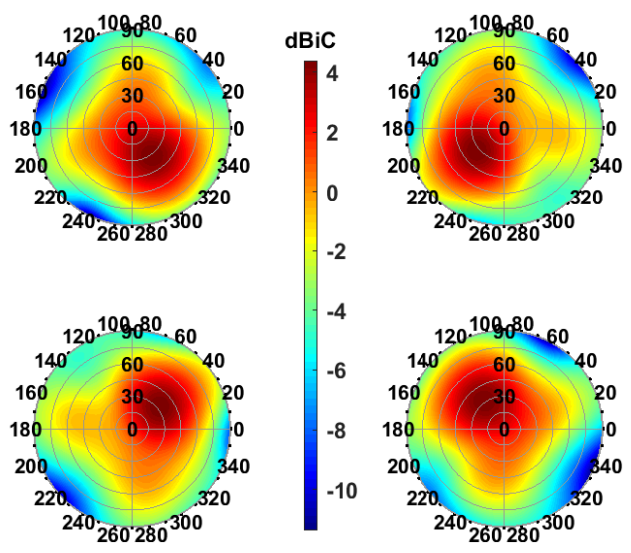

Fig. 4. The simulated realized gain patterns, $|\mathbf{h}(\theta, \phi)|$.

\section{B. Objective Array}

We propose a three step approach for the determination of an appropriate response for the objective array. The starting point is to provide an initial array response matrix named $\stackrel{\circ}{H}_{A}$. Second, a numerical optimization delivers an $\stackrel{\circ}{H}_{B}$ matrix. Third, this $\stackrel{\circ}{H}_{B}$ matrix is averaged to standardize the $N$ element array to an equally behaved and uniformly distributed AA, which leads to the objective response matrix $\stackrel{\circ}{H}$. Schematically, the sequence is,

$$
\text { 1) } \left.\stackrel{\circ}{H}_{A} \longrightarrow \text { 2) } \stackrel{\circ}{H}_{B} \longrightarrow 3\right) \stackrel{\circ}{H}
$$

1) Initial objective array response matrix $\stackrel{\circ}{H}_{A}$ : A virtual array constrained to behave like equal and uncoupled antennas has an array manifold whose $N$ rows are identical about their magnitude. In addition, considering the diagonal components of (5) and (6), they can only be equal if the amplitude of the gain patterns at each of the selected DOA's is equal to the root mean square of the actual AA response. Then, the magnitude of the elements of the $\stackrel{\circ}{H}_{A}$ matrix must be,

$$
\stackrel{\circ}{R}_{p, p}=R_{p, p} \Rightarrow\left|\stackrel{\circ}{h}_{n, p}^{A}\right|=\sqrt{\frac{1}{N} \sum_{m=1}^{N}\left|h_{m, p}\right|^{2}},
$$

for all the virtual antennas $n=1, \ldots, N$ at every DOA in the set $p=1, \ldots, P$. While for the phase characteristics, a good initial guess is that they match the relative phases associated to the actual locations of the antenna elements. Then, its $N \times P$ components are proposed to be,

$$
\angle h_{n, p}^{A}=\frac{2 \pi d_{n}}{\lambda} \sin \left(\theta_{p}\right) \cos \left(\phi_{p}-\varphi_{n}\right),
$$

where $d_{n}=d / \sqrt{2}=\lambda /(3 \sqrt{2})$ and $\varphi_{n}=\varphi_{\text {ref }}-2 \pi \frac{(n-1)}{N}$ are the polar coordinates of each antenna relative to the geometric center of the array, and $\varphi_{\text {ref }}=3 \pi / 4$ is the reference angle that matches the first antenna orientation.

2) Optimized objective array response matrix $\stackrel{\circ}{H}_{B}$ : Considering (8), the remaining parameters for optimization are the phase components of $H_{A}$ that provide a number of $N \times P$ degrees of freedom that allow us to satisfy the minimization problem (7). However, the Frobenius norm depends on a total of $P \times P$ terms, where $P \gg N$ is commonly the case. Consequently, this leads to a numerical optimization problem that can be solved by the Steepest Descent Method (SDM) [25]. Then, the following iterative process takes place by starting from the $\stackrel{\circ}{H}_{A}$ matrix:

1) Initialize $\stackrel{\circ}{H}_{B}=\stackrel{\circ}{H}_{A}, \stackrel{\circ}{R}_{B}=\stackrel{\circ}{H}_{B}^{H} \stackrel{\circ}{H}_{B}, \gamma_{B}=\left\|R-\stackrel{\circ}{R}_{B}\right\|_{F}^{2}$, and the step size $\alpha$, of the iterative SDM.

2) The gradient of $\gamma_{B}$ about the phase components of $\stackrel{\circ}{H}_{B}$ is the matrix $\delta$ that is computed by,

$$
\delta_{n, p}=4 \Re\left\{\sum_{q=1}^{P}\left[j \stackrel{\circ}{h}_{n, p} \stackrel{\circ}{h}_{n, q}^{*}\left(\stackrel{\circ}{r}_{p, q}-r_{p, q}\right)\right]\right\} .
$$

3) Update the phase components in the opposite direction of the gradient with step $\alpha$,

$$
\angle \stackrel{\circ}{H}_{B}=\angle \stackrel{\circ}{H}_{B}-\alpha \delta,
$$

and then the correlation $\stackrel{\circ}{R}_{B}$ and the cost function $\gamma_{B}$.

4) If an acceptable low value of $\gamma_{B}$ or other stopping criteria are still not met, verify the convergence of $\gamma_{B}$, update $\alpha$ if necessary, and then return to step 2).

This approach aims to achieve the optimum array response $\stackrel{\circ}{H}_{B}$ that maximizes the correspondence between the $R$ and $\stackrel{\circ}{R}_{B}$ matrices. One possible drawback is that the $\gamma$ function may not be necessarily unimodal/quasiconvex. If so, there could be several local minima, and then the convergence to a global minimum will not be guaranteed [25]. However, the initial matrix $H_{A}$ is a good starting point, and with proper control of the speed convergence, a useful solution can be obtained.

3) Objective array response matrix $\stackrel{\circ}{H}$ : The matrix $\stackrel{\circ}{H}$ will be described in terms of its gain and steering matrices. The objective gain patterns should be equal for all the antennas, thus it incorporates the common factor of $\stackrel{\circ}{H}_{B}$. While the objective steering matrix will be an approximation of the remaining phase variations. This approximation consists on assuming $N$ equally behaved antennas and $2 \pi / N$ rotated one from the other, which is done for complexity reduction of the fitted steering vector, that will be presented in Section III.E.

The amplitudes of the gain matrix $\stackrel{G}{ }$ remain unchanged from (8), while the phases are obtained after averaging the individual RP's to extract the common phase term. Hence,

$$
\left|\stackrel{\circ}{g}_{n, p}\right|=\left|\stackrel{\circ}{h}_{n, p}^{B}\right|, \quad \angle \stackrel{\circ}{g}, p_{n, p}=\frac{1}{N} \sum_{m=1}^{N} \angle \stackrel{\circ}{h}_{m, p}^{B},
$$

for $n=1, \ldots, N$ and $p=1, \ldots, P$. Next, the remaining variations of the phase components of $\stackrel{\circ}{H}_{B}$ are incorporated to the $\stackrel{S}{S}$ matrix as follows,

$$
\begin{aligned}
\angle \stackrel{s}{s}_{1}\left(\theta_{p}, \phi_{p}\right)= & \frac{1}{N} \sum_{m=1}^{N} \psi_{m}\left(\theta_{p}, \phi_{p}+2 \pi(m-1) / N\right), \\
& \angle \stackrel{s}{s}_{n+1}\left(\theta_{p}, \phi_{p}\right)=\angle \stackrel{s}{s}_{n}\left(\theta_{p}, \phi_{p}+2 \pi / N\right),
\end{aligned}
$$

for $p=1, \ldots, P$ and $n=1, \ldots, N-1$, where $\psi_{m}\left(\theta_{p}, \phi_{p}\right)=$ $\angle \stackrel{\circ}{h}_{m, p}^{B}-\angle \stackrel{\circ}{g}_{m, p}$.

That yields the averaged array response matrix, 


$$
\stackrel{\circ}{H}=\stackrel{\circ}{S} \circ \stackrel{\circ}{G} .
$$

This matrix defines the radiation properties of an ideal array with identically behaved elements at the set of $P$ DOA's. It is the proposed matrix to minimize the cost function $\gamma$ in (7).

\section{Orthonormal Transformation}

Since the matrices $H$ and $\stackrel{\circ}{H}$ are already defined, the search for the unitary matrix $W$ is immediate. The closed-form solution for $W$ is,

$$
W=U V^{H},
$$

expressed in terms of the unitary matrices $U$ and $V$ obtained from the Singular Value Decomposition of ${ }^{H} Q Q^{H} H^{H}=$ $U \Sigma V^{H}$ [18]. $Q$ is an optional weight matrix that could be used for prioritizing the quality of the transformation in some directions over others. In this work, it has been taken as the identity matrix. Consequently, we obtain the compensated matrix $\tilde{H}$ as,

$$
W H=\tilde{H} \approx \stackrel{\circ}{H} .
$$

The approximate equality comes from the fact that the virtual array might not perfectly meet the imposed conditions of having equally behaved elements or being rotationally symmetric. However, the smaller the magnitude of the $\gamma$ function, the closer $\tilde{H}$ will be to $\stackrel{\circ}{H}$.

Even though the whole process described previously to find the virtual array is computationally intensive, it should be noted that it is supposed to be done only once and offline, unless a complete AA actual RP's recalibration will be considered. Afterwards, the AA is endowed with a unitary matrix $W$ of size $N \times N$, and a function that describes the virtual steering vector $\stackrel{\circ}{\mathbf{s}}$, which in our case depends on only two parameters, as it will be shown in the Section III.E.

In particular, the unitary matrix for our designed AA was,

$$
\begin{aligned}
& W=\left[\begin{array}{ll}
0.9429 e^{-j 3.1069} & 0.2286 e^{+j 1.7479} \\
0.2392 e^{-j 1.8484} & 0.9440 e^{+j 0.0442} \\
0.1099 e^{+j 1.1305} & 0.2160 e^{+j 1.4695} \\
0.2042 e^{-j 1.6102} & 0.0998 e^{-j 2.0530}
\end{array}\right.
\end{aligned}
$$

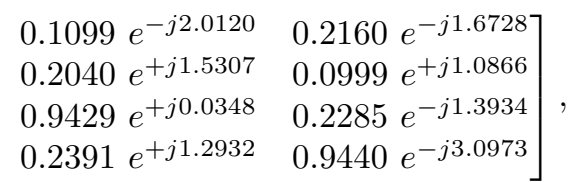

that yields to the virtual array response. The magnitude of their gain patterns are presented in Fig. 5.

\section{Compensation Assessment}

Many other compensation methods are based on a least mean squares approach (LMS) where the transformation matrices are not restricted to be unitary [16], [17]. Thus, when searching for a transformation matrix these methods can make use of more degrees of freedom than in ours. In the following, we compare the transformation error between both proposals with the following normalized error function [26]:

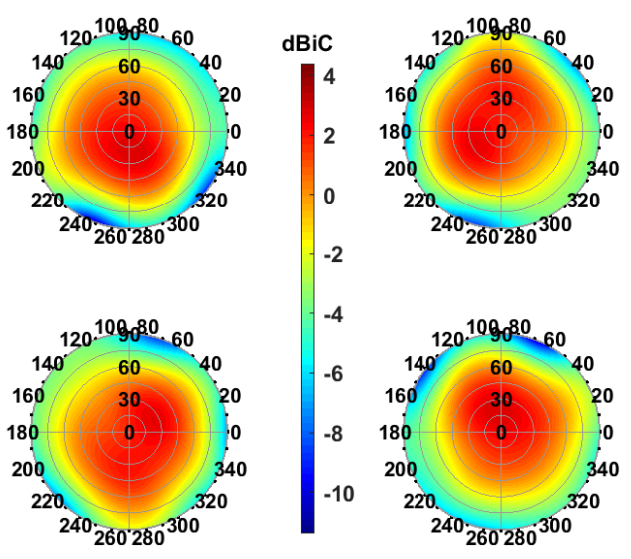

Fig. 5. The virtual AA realized gain patterns, $|\tilde{\mathbf{h}}(\theta, \phi)|$.

$$
\Gamma(\stackrel{\circ}{H})=\frac{\|\stackrel{\circ}{H}-C H\|_{F}^{2}}{\|\stackrel{\circ}{H}\|_{F}^{2}}
$$

being $C$ the compensation unitary matrix $W$, or the LMS transformation matrix $T$ that is obtained through:

$$
T=\stackrel{\circ}{H} H^{T}\left(H H^{T}\right)^{-1} \quad \text { s.t. } \quad T H \approx \stackrel{\circ}{H}
$$

The $\Gamma$ errors are compared for the objective matrix $\stackrel{\circ}{H}$, and are presented at the last column of Table II. It is seen that in this case there are no significant differences between both approaches. In fact, we found that $W$ and $T$ are quite similar, which means that the designed AA and the developed virtual array leads the LMS approach to yield an almost unitary matrix. Additionally, the $\Gamma$ errors are also calculated for the objective matrices $\stackrel{\circ}{H}_{A}$ and $\stackrel{\circ}{H}_{B}$ to demonstrate the effectiveness of the proposed objective array response. Each of them require their own transformation matrices that are obtained with Eqs. (16) and (20). The results are also presented in Table II. It can be observed that the errors for the initial objective matrix $\stackrel{\circ}{H}_{A}$ become significantly higher for both methods, and that the difference in performance between the unitary and the LMS transformations grows in favor of the LMS. This is because the initial objective matrix represents an array response that is not as suitable to be achieved with a unitary matrix. The transformation error is significantly reduced when the optimized array response matrix $H_{B}$ is used. Finally, given the phase response structure constraints used to obtain the objective array response $\stackrel{H}{H}$, the error is slightly increased compared to the previous case.

TABLE II

TRANSFORMATION ERRORS WITH UNITARY AND LMS MATRICES.

\begin{tabular}{|c|c|c|c|}
\hline Approach / Transf. Error & $\Gamma\left(\stackrel{\circ}{H}_{A}\right)$ & $\Gamma\left(\stackrel{\circ}{H}_{B}\right)$ & $\Gamma(\stackrel{\circ}{H})$ \\
\hline Unitary matrix $(W)$ & 0.0313 & 0.0081 & 0.0093 \\
\hline LMS matrix $(T)$ & 0.0171 & 0.0078 & 0.0088 \\
\hline
\end{tabular}

\section{E. Fitted Steering Vector $\stackrel{s}{\mathbf{s}}(\theta, \phi)$}

The $\stackrel{G}{G}$ matrix is no longer relevant at this stage since it can be seen as a common factor during array processing. Hence, 
the search for a fitting function of the steering vector $\stackrel{\mathbf{s}}{\mathbf{S}}(\theta, \phi)$ is intended to resemble as much as possible the phase variations of the $\stackrel{\circ}{S}$ matrix. In this way, a GNSS receiver can effectively manage the AA with this function without the need of working with look-up tables for the RP's phases. This function will be restricted in order to satisfy the following conditions:

- $\stackrel{\mathbf{s}}{(} \theta, \phi)$ must be a bijective function to generate an unambiguous correspondence between $\mathbf{h}(\theta, \phi)$ and $\mathbf{h}(\theta, \phi)$ manifold vectors to avoid any disagreement or loss of generality in DOA discrimination. Luckily, this is ensured when their correlation functions are similar enough.

- The $\stackrel{\mathbf{s}}{(}(\theta, \phi)$ vector is restricted to accomplish a rotational symmetry: $\stackrel{\circ}{s+1}_{n+1}(\theta, \phi)=\stackrel{\circ}{s}_{n}(\theta, \phi+2 \pi / N), n=1, \ldots, N-$ 1 . It implies $2 \pi / N$-rotated, equally behaved antennas.

Therefore, our proposal simply consist of a steering vector that considers the phase differences as a consequence of different traveling-wave path lengths. The rotational symmetry of this virtual array, that we have already adopted in Section III.C, implies two fitted parameters: antennas located at a distance $a_{1}$ from the origin and aligned so that the first antenna is oriented at the reference angle $a_{2}$. The geometry of this array is described by the following steering vector,

$$
\stackrel{\circ}{s}_{n}(\theta, \phi)=e^{j 2 \pi a_{1} \sin (\theta) \cos \left(\phi-a_{2}+2 \pi \frac{n-1}{N}\right)},
$$

with $n=1,2, \ldots, N$. In our particular array, the minimum squared fitting parameters were found to be $a_{1}=0.2719$ and $a_{2}=2.509$. This function is similar to (9) with the peculiarity of having greater distance between antennas, $a_{1}>d /(\lambda \sqrt{2})$, and a slight rotation of the array orientation, $a_{2} \gtrsim \varphi_{\text {ref }}$. It is remarkable that the differences between the actual RP's that are induced by the electromagnetic coupling are actually translated into an ideal AA with higher resolution given its larger aperture [18], [27].

\section{Simulated Performance}

The use of an AA provides the possibility of steering the beam/s of its combined radiation pattern and allows maximizing the gain towards the DOA of an impinging signal [15]. Acceptable results are frequently obtained even if the true DOA's of the satellites or the AA calibration information are not well known. On the contrary, when the objective is to minimize the received power that comes from an unwanted source, this information is essential for good performance of deterministic nullforming techniques [28], [29]. This is typically the case when an in-band undesired wave is also impinging on the AA of a GNSS receiver. Hence, it is important to verify if an improvement can be achieved with our approach for array processing when no information about actual/calibrated RP's is used. Instead, only the relative antenna phase differences are supposed to be deduced from their relative positions.

Here we present a simulated interference mitigation experiment employing the AA described above. The test consists of comparing the performance of the AA to overcome a RHCP continuous-wave jamming attack operating at the center frequency of the L1 GPS band $f_{c}$, while enhancing the AA gain at the DOA of a GPS signal. Given the characteristics of the signals and the assumed interference spectra, a narrowband model will be used for signal processing, and $f_{c}$ will be taken as the reference frequency. It also will be assumed that any interference wave incomes from the near-horizon region, while the usable GPS signals are likely closer to the zenith angle, assuming the zenith orientation to be normal to the plane array. For the sake of simplicity, only one GPS satellite signal of interest is considered arriving from $\left(\theta_{s}, \phi_{s}\right)$, while an undesired source signal arrives from $\left(\theta_{i}, \phi_{i}\right)$, according to the values in Table III.

TABLE III

DOA'S OF INTEREST FOR THE SIMULATION SCENARIO.

\begin{tabular}{|c|c|}
\hline Desired DOA & Undesired DOA \\
\hline$\theta_{s}=0^{\circ}$ & $\theta_{i}=50^{\circ}, 70^{\circ}$ or $90^{\circ}$ \\
$\phi_{s}=0^{\circ}$ & $0^{\circ} \leq \phi_{i}<360^{\circ}$ \\
\hline
\end{tabular}

The overlapping snapshots of the GPS signal $s$, the interference $i$ and the thermal noise $\mathbf{n}$ that are received by the AA, are considered in the array signal vector $\mathbf{x}$ :

$$
\mathbf{x}=s \mathbf{h}\left(\theta_{s}, \phi_{s}\right)+i \mathbf{h}\left(\theta_{i}, \phi_{i}\right)+\mathbf{n} .
$$

Hence, the beamformer yields the array output signal $y$, that is a linear combination of the vector $\mathbf{x}$ and the complex array weight vector $\mathbf{c}$ :

$$
y=\mathbf{c}^{H} \mathbf{x} .
$$

In essence, a new RP is obtained through beamforming [15]:

$$
h_{\mathrm{BF}}(\theta, \phi)=\mathbf{c}^{H} \mathbf{h}(\theta, \phi) .
$$

Therefore, given the signals of interest, we define the gain ratio between the jammer to GPS satellite DOA's as a figure of merit for the comparisons. Namely,

$$
\kappa=\left|\frac{h_{\mathrm{BF}}\left(\theta_{i}, \phi_{i}\right)}{h_{\mathrm{BF}}\left(\theta_{s}, \phi_{s}\right)}\right| .
$$

Hereafter, in order to emulate an on-board scheme, the spatial processing is done by using just the phased array model. Afterwards, the performance analysis is done with all the available information.

\section{A. Fully Controlled Scenario}

In this first comparison, the orientation of the array is assumed to be known, as well as the GPS signal and interference DOA's. The Orthogonal Projection Beamforming (OPB) is then used to obtain the proper weight vectors [15].

The first case considered is simply an uncompensated approach (labeled as "UA") that assumes an idealistic array of isotropic elements. It will be compared against the compensation schemes at a similar level of computational complexity at the signal processing stage, i.e. the beamformer weights are calculated only using the steering vectors $s_{n}(\theta, \phi)$ and not accounting for the antenna gains $g_{n}(\theta, \phi)$ :

$$
\mathbf{c}=\left[I_{N}-\left(\mathbf{s}_{i}\left(\mathbf{s}_{i}^{H} \mathbf{s}_{i}\right)^{-1} \mathbf{s}_{i}^{H}\right)^{H}\right] \mathbf{s}_{s},
$$


being $\mathbf{s}_{s}=\mathbf{s}\left(\theta_{s}, \phi_{s}\right)$ and $\mathbf{s}_{i}=\mathbf{s}\left(\theta_{i}, \phi_{i}\right)$, see (1).

The second case considered uses LMS compensation ("LC"), whose transformation matrix $T$ is based on the initial objective array $\stackrel{\circ}{H}_{A}$ since, as usually done, it preserves the original array geometry. Hence, the OPB also yields to (26).

The last case is our unitary compensation ("UC") method based on the matrix $W$ associated to the virtual array model $\stackrel{\circ}{H}$. Then, its OPB weight vector $\stackrel{\circ}{\mathrm{c}}$ is,

$$
\stackrel{\circ}{\mathbf{c}}=W^{H}\left[I_{N}-\left(\stackrel{\circ}{\mathbf{s}}_{i}\left(\stackrel{\circ}{\mathbf{s}}_{i}^{H} \stackrel{\circ}{\mathbf{s}}_{i}\right)^{-1}{\stackrel{\circ}{\mathbf{s}_{i}^{H}}}^{H}\right)^{H}\right] \stackrel{\circ}{\mathbf{s}}_{s},
$$

being $\stackrel{\circ}{\mathbf{s}}_{s}=\stackrel{\circ}{\mathbf{s}}\left(\theta_{s}, \phi_{s}\right)$ and $\stackrel{\circ}{\mathbf{s}}_{i}=\stackrel{\circ}{\mathbf{s}}\left(\theta_{i}, \phi_{i}\right)$.

The graphs in Fig. 6 present the calculated gain ratios $\kappa$ when the incidence angle of the interference is swept as indicated in Table III. The results of the uncompensated AA, the LMS compensated AA, and the unitary compensated AA are labeled as $\kappa^{(\mathrm{UA})}, \kappa^{(\mathrm{LC})}$ and $\kappa^{(\mathrm{UC})}$ respectively. The performance of the OPB in the compensated cases is significantly improved in most part of the hemisphere, reaching gain ratios in the order of $-20 \mathrm{~dB}$ or less when $\theta_{i}=50^{\circ}$. Hence, without compensation the interference attenuation level is not effective when the interference approaches the zenith region. Both compensation methods provide good results in this region, presenting only slight differences according the DOA.

For the particular case where the interferences come from the horizon, neither case produces a significant improvement, being the natural antenna gain reduction the main contributor to the interference attenuation. If a better behaviour would be needed, it is possible to use a different weight matrix $Q$ to enhance the performance of this region, at the cost of some degradation in the upper region (see Section III.C). Another possible approach could be to adopt an alternative sectorial transformation, instead of the global one, to take over of a narrower DOA region when required.

\section{B. Partially Controlled Scenario. Deterministic Beamforming.}

Now, the jammer position is assumed to be unknown, so it will be necessary first to detect its position by employing a direction finding method. We chose the MUSIC algorithm since it is a very well known method and it can incorporate some level of information about the RP's [30]. It is based on the sampled spatial correlation matrix $R_{\mathrm{xx}}$ of the received signals $\mathbf{x}$. Since the GPS uses direct sequence spread spectrum modulation, their signals are immersed well below the noise power level, thus without despreading they are undetectable [1]. Therefore, before the despreading a strong interference can be easily detected, since the MUSIC algorithm produces a DOA estimate by searching the array response vector that is orthogonal or nearly orthogonal to the noise subspace. Namely, the estimation yields $\left(\hat{\theta}_{i}, \hat{\phi}_{i}\right) \approx\left(\theta_{i}, \phi_{i}\right)$, but the results will be different depending on the selected compensation approach.

Given the stochastic nature of this problem, it is convenient to define a more concrete scenario. The signal and noise power densities are selected to be the typical values [1], while for the interference a generic but realistic value is adopted. The models of the RF front-end and the analogto-digital converters are fully simplified, but some relevant characteristics are taken from the heterodyne receiver that will be used at the experimental validation stage, i.e.:

- Intermediate frequency: $f_{I F}=30.42 \mathrm{MHz}$.

- Sampling frequency: $f_{s}=20 \mathrm{MHz}$.

- Bandwidth: $B W=12 \mathrm{MHz}$.

- Number of samples: 400000.

- Antenna noise power density: $N_{0}=-174 \mathrm{dBm} / \mathrm{Hz}$.

- GPS Carrier to noise power density: $C / N_{0}=40 \mathrm{~dB}-\mathrm{Hz}$.

- Interference-to-signal ratio: $I S R=40 \mathrm{~dB}$.

The DOA estimation results are shown in polar graphs in Fig. 7. The calibrated array solutions (black dotted curves) are shown only for reference purposes since they require access to the whole array response information. The other curves indicated as $\left(\hat{\theta}_{i}, \hat{\phi}_{i}\right)^{(\mathrm{UA})},\left(\hat{\theta}_{i}, \hat{\phi}_{i}\right)^{(\mathrm{LC})}$ and $\left(\hat{\theta}_{i}, \hat{\phi}_{i}\right)^{(\mathrm{UC})}$ represent the solutions of the uncompensated AA model, the LMS compensated AA model and the unitarily compensated virtual AA model respectively. The dashed curves with symbols present the solutions of the already defined scenario. In this case, the LMS and the unitary compensated arrays provide very good results in most part of the hemisphere. When approaching the horizon, there are two almost bounded sectors where neither non-calibrated solutions are consistent with the true DOA's. In order to obtain further improvement, they would need to be treated differently since they disagree due to the relatively low antenna gains at that sectors. This evidence is in accordance with the results of the previous subsection, so the same conclusions also apply for a possible improvement of the DOA estimation. Nevertheless, it can be seen that our approach shows slightly better results, even in this relatively low noise context.

Since the LMS approach alters the noise correlation structure, its weakness can be evidenced with a higher noise power level. Lets suppose then a more adverse case where the noise power density equals to $N_{0}=-164 \mathrm{dBm} / \mathrm{Hz}$, i.e., an increase of $10 \mathrm{~dB}$, which implies a $C / N_{0}$ of $30 \mathrm{~dB}-\mathrm{Hz}$ that can be considered close to the lowest useful signal level in a typical GPS receiver. The dotted curves with symbols present the solutions of this new scenario. Only the LMS solutions have changed considerably showing high deviations from the true DOA's. On the contrary, the use of a unitary matrix effectively retains the advantages of applying a compensation, while also avoids the distortion of the received noise properties. It is important to notice that any other estimation methods that assume a phased array model and/or do not account for the RP's, can also benefit from this transformation [31].

Based on the interference DOA estimations, the OPB weight vectors (26) and (27) are recalculated correspondingly. Hence, the results in terms of the gain ratios as a function of $\left(\theta_{i}, \phi_{i}\right)$ are presented in Fig. 8. These are consistent with the results found in the previous subsection. In many cases, the differences obtained with the DOA estimations (errors) even improve the $\kappa$ ratio since they conform to irregularities of the actual RP's. However, this is not the case when considering the LMS compensation with relatively high noise power level because of the bias produced in the MUSIC method by a correlated noise subspace. 


\section{Partially Controlled Scenario. Adaptive Beamforming.}

Typically, the spatial subspace projection based on the DOA estimation is applied for mitigation of spoofing/meaconing attacks, while for the interference/jamming problem an adaptive subspace projection process is preferred [7], [8], [13]. Adaptive beamforming is commonly chosen because it often requires very little information about the array response. However, there are situations where a rough model for unequally behaved antennas can also induce some signal degradation. In this subsection, we show that applying a compensation can improve the performance in these situations and that our approach can produce better results than LMS.

Here, we use the Minimum Variance Distortionless Re- sponse (MVDR) beamforming method [15]. It performs a blind attenuation of an interference, based on the sampled spatial correlation matrix $R_{\mathrm{xx}}$, while recovering the desired signal by maximizing the gain towards its location. Hence, the MVDR estimators for the three cases are,

$$
\begin{aligned}
y^{(U A)}=\mathbf{c}_{1}^{H} \mathbf{x}, & \mathbf{c}_{1}=R_{\mathbf{x x}}^{-H} \mathbf{s}_{s}, \\
y^{(L C)}=\mathbf{c}_{2}^{H} \mathbf{x}^{(L C)}, & \mathbf{c}_{2}=R_{\mathbf{x}^{(L C)} \mathbf{x}^{(L C)}}^{-H} \mathbf{s}_{s}, \\
y^{(U C)}=\mathbf{c}_{3}^{H} \mathbf{x}^{(U C)}, & \mathbf{c}_{3}=R_{\mathbf{x}^{(U C)} \mathbf{x}^{(U C)}}^{-H} \stackrel{\circ}{s}_{s}
\end{aligned}
$$

where $R_{\mathbf{x}^{(L C)} \mathbf{x}^{(L C)}}$ and $R_{\mathbf{x}^{(U C)} \mathbf{x}^{(U C)}}$ are the sampled correlation matrices of the transformed array signals $\mathbf{x}^{(L C)}=T \mathbf{x}$ and $\mathbf{x}^{(U C)}=W \mathbf{x}$ respectively, while $y^{(U A)}, y^{(L C)}$ and $y^{(U C)}$ are the combined array signals. With this method, a compensation

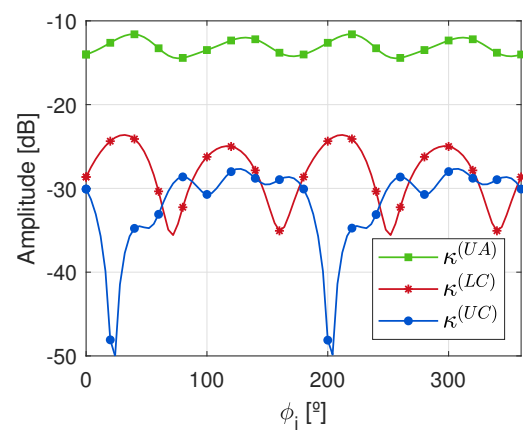

(a) $\theta_{i}=50^{\circ}$

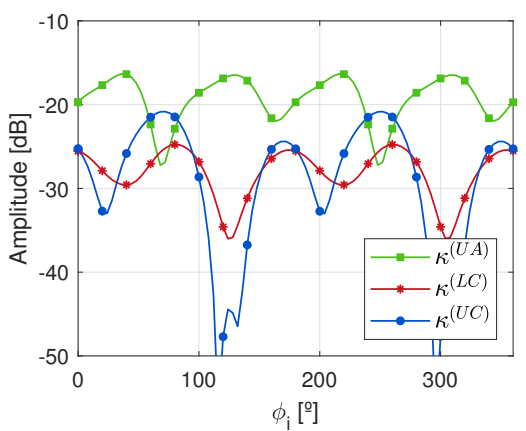

(b) $\theta_{i}=70^{\circ}$

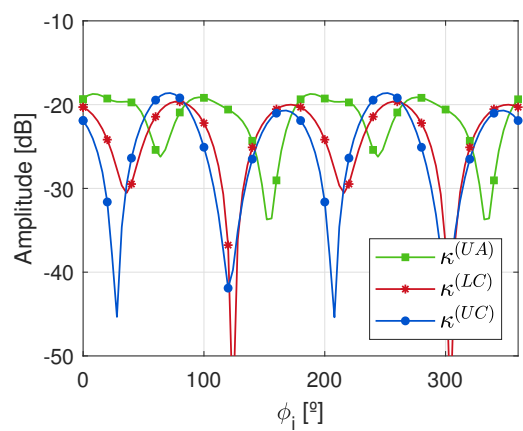

(c) $\theta_{i}=90^{\circ}$

Fig. 6. Comparison of the interference gain to signal gain rejection ratio $(\kappa)$ after beamforming for different interference DOA's.

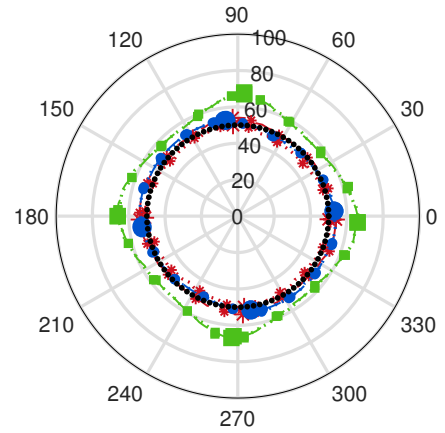

(a) $\theta_{i}=50^{\circ}$

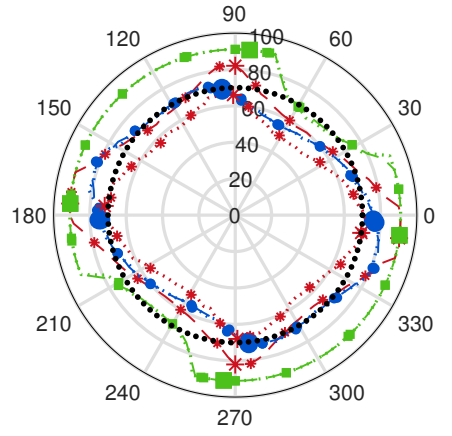

(b) $\theta_{i}=70^{\circ}$

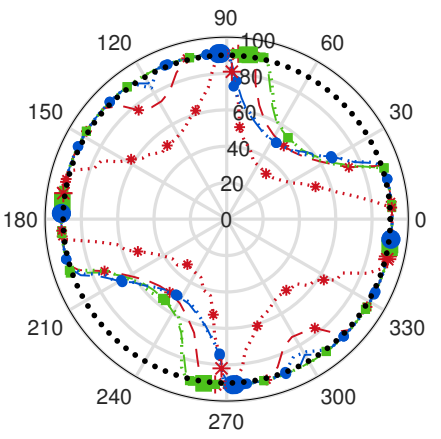

(c) $\theta_{i}=90^{\circ}$

Fig. 7. DOA of the interference estimations. Calibrated AA (dots), Uncomp. AA (squares), LMS Comp. AA (asterisks), Unit. Comp. AA (circles). At the legend, one star $(\star)$ symbolizes the relatively low noise case, and two stars $(\star \star)$ symbolize the high noise case. The curves $\left(\hat{\theta}_{i}^{\star}, \hat{\phi}_{i}^{\star}\right)^{(U A)}$ compared to $\left(\hat{\theta}_{i}^{\star \star}, \hat{\phi}_{i}^{\star \star}\right)^{(U A)}$, and $\left(\hat{\theta}_{i}^{\star}, \hat{\phi}_{i}^{\star}\right)^{(U C)}$ compared to $\left(\hat{\theta}_{i}^{\star \star}, \hat{\phi}_{i}^{\star \star}\right)^{(U C)}$ are quite similar and appear overlapped.

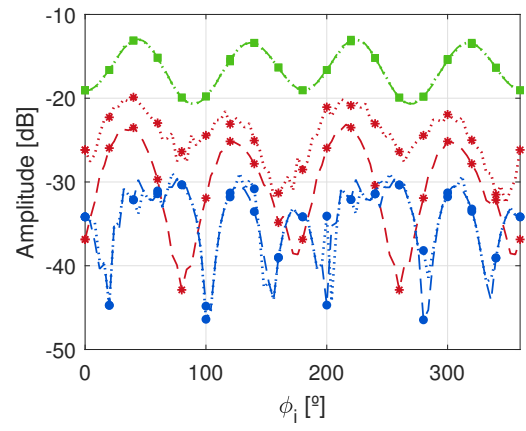

(a) $\theta_{i}=50^{\circ}$

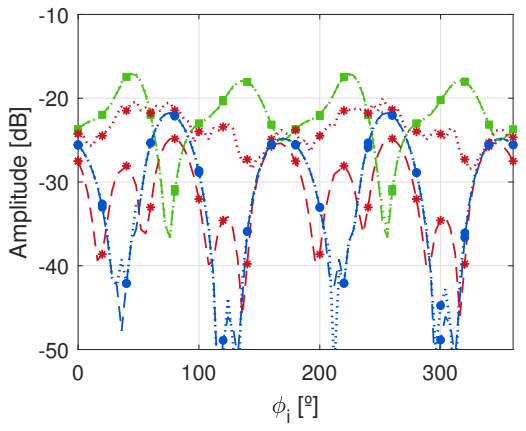

(b) $\theta_{i}=70^{\circ}$

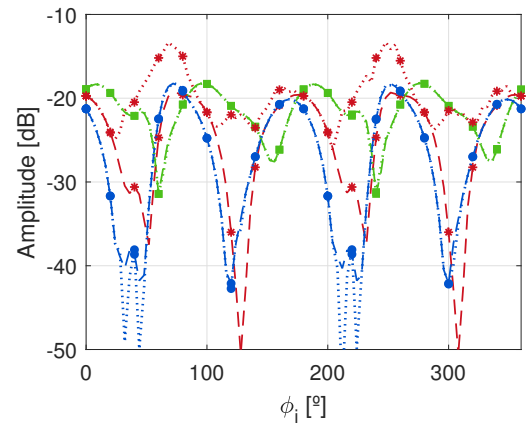

(c) $\theta_{i}=90^{\circ}$

Fig. 8. Comparison of $\kappa$ for different interference DOA's using MUSIC estimates. 
does not make a significant contribution to the mitigation purpose, so the ratio $\kappa$ does not show substantial differences. However, since the transformation allows to incorporate more information of the actual AA structure, we show that some improvement can be obtained in the final carrier power to noise power density ratio, $C / N_{0}$.

The same scheme of the previous examples -without increasing the noise level- is used, with the only difference that the desired signal comes from $\left(60^{\circ}, 180^{\circ}\right)$. For the interference located at $\theta_{i}=90^{\circ}$ and $0^{\circ} \leq \phi_{i}<360^{\circ}$, the $C / N_{0}$ of the already defined cases are shown in Fig. 9. The calibrated AA solution is again included as an upper limit reference, so the difference in $C / N_{0}$ with respect to each method, $\Delta C / N_{0}$, eases the comparison. The weight vector of this calibrated array ("CA") is $\mathbf{c}_{4}=R_{\mathbf{x x}}^{-H} \mathbf{h}_{s}$, which yields to $y^{(C A)}=\mathbf{c}_{4}^{H} \mathbf{x}$. It is evident that the performance of the AA with unitary compensation is practically as good as the calibrated case, while the other approaches lose some signal strength, in greater proportion when the interference and the desired signal are closer.

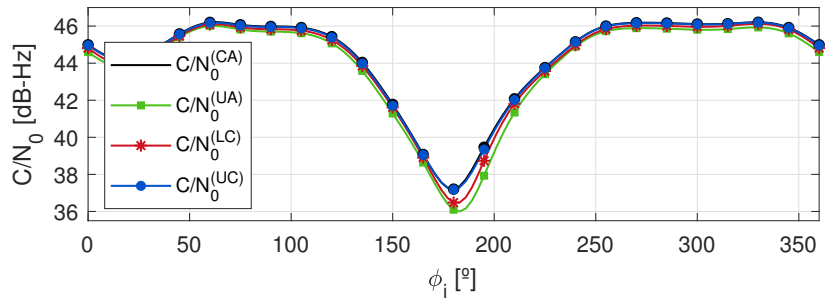

(a) $C / N_{0}$ of the four approaches. CA taken as upper limit.

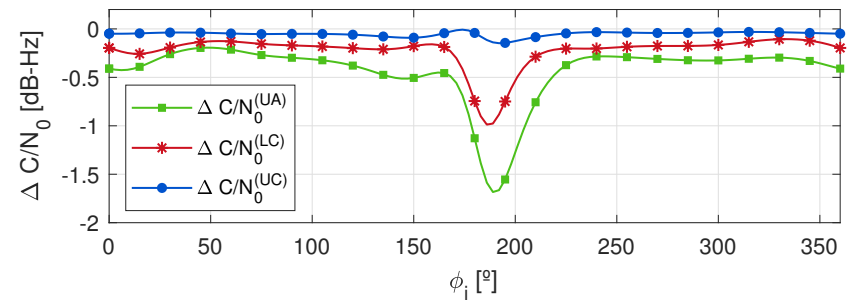

(b) Relative $C / N_{0}$ with respect to the calibrated AA performance.

Fig. 9. MVDR performance when an interference is present at $\theta_{i}=90^{\circ}$ and a satellite signal is coming from $\left(60^{\circ}, 180^{\circ}\right)$.

\section{IMPLEMENTATION AND MEASUREMENTS}

The four-element AA prototype was constructed according the specifications described in Section III.A. Additionally, a thermoplastic polymer based structure was added to carry out its mounting. This holder has small flaps that allow the AA to be fitted in through its corners, that are small enough to not interfere with the designed array response. The implemented AA with its holder are shown in Fig. 10.

The measured scattering parameters, presented in Fig. 11, are consistent with the simulations in Fig. 3. Although there are some minor differences, we verified that the AA performance is not significantly affected.

The AA under test was validated using a multi-antenna GNSS experimental receiver of our own design, with similar architecture to the receiver in [32]. The signals of each antenna

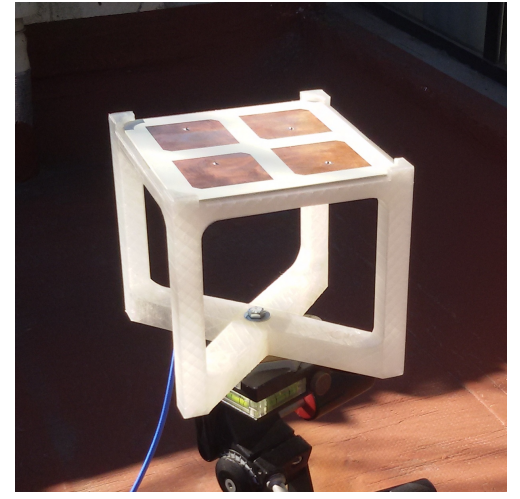

Fig. 10. The antenna array mounted over the thermoplastic holder.

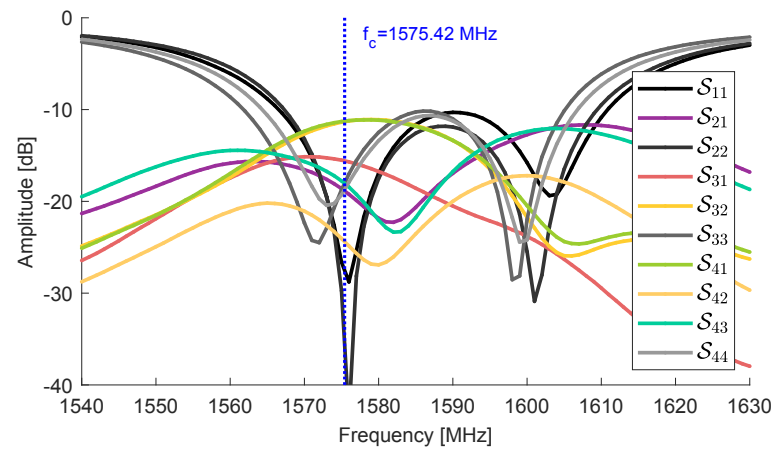

Fig. 11. Measured scattering parameters.

were recovered after passing through the RF front-end stage and then digitized at the intermediate frequency, $f_{I F}$. The data acquisition stage uses a high speed and high bandwidth sampling and storage platform whose architecture is based on [33]. The main parameters of the receiver used in this test are,

- Local oscillator: $1545 \mathrm{MHz}$

- Reference oscillator (OCXO): $10 \mathrm{MHz}$

- Intermediate frequency: $f_{I F}=30.42 \mathrm{MHz}$.

- Sampling frequency: $f_{s}=20 \mathrm{MHz}$.

- Bandwidth: $B W=12 \mathrm{MHz}$.

- Quantization: 2 bit per channel.

- Number of registered samples: 400000.

The receiver and the AA were located in an open-sky environment, as depicted in Fig. 12, in order to acquire $20 \mathrm{~ms}$ of GPS data samples. Afterwards, the data was processed in a computer for synchronization with GPS signals. The time and place of the experiment was December 10th of 2019 at 13:35 hs, in the city of La Plata, Buenos Aires, Argentina. Eight satellites above an elevation mask of $10^{\circ}\left(\theta \leq 80^{\circ}\right)$ were available and all of them were detected at each of the antennas. The satellites in view, labeled by their Space Vehicle Numbers (SVN's), were 2, 5, 12, 21, 25, 26, 29, and 31.

Next, we analyzed the DOA estimation and nullforming capabilities of the AA, as we did by simulation. Here, the calibrated case is not considered since we did not have measured the RP's. The LMS compensation case is neither included because the performance results that were obtained are quite similar to the ones obtained with our method. In fact, a slight but consistent improvement was found with this last 
approach, in the range from 0 to $1 \mathrm{~dB}$. As a general statement, we can say that we have found a good agreement between experimental results and previous simulations, and therefore the implementation has been successful.

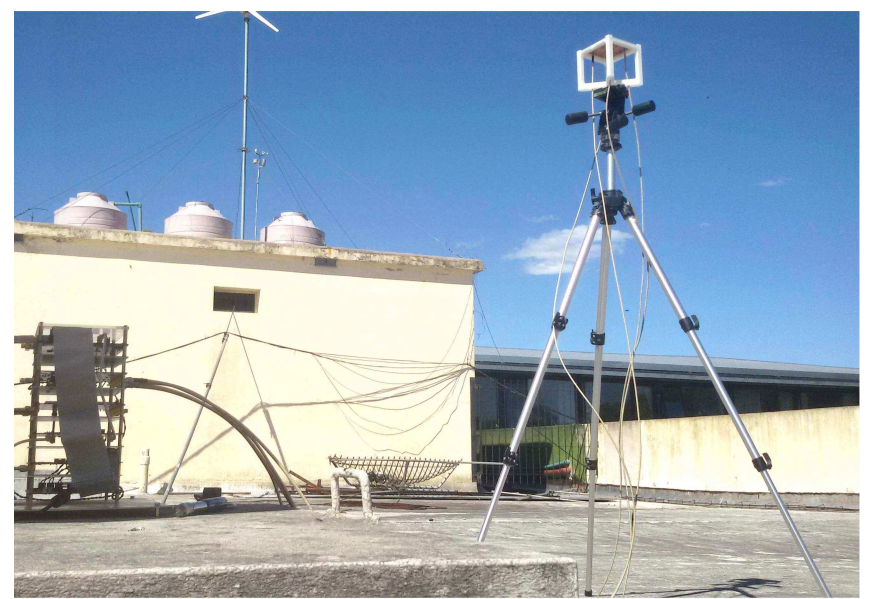

Fig. 12. Test environment.

The polar graph in Fig. 13 shows the DOA's of the satellites in view. It can be evidenced that there are several satellites with low and high elevation angles spread in the range of $0^{\circ}<\theta<$ $90^{\circ}$. The virtual AA model validation for DOA of the satellites estimation was done with the MUSIC algorithm. Given that the GPS satellites use spread spectrum code division multiple access [1], the algorithm was applied after the despreading stage for each satellite at a time. The results obtained with and without compensation are also illustrated in Fig. 13. In both cases, a good correspondence is obtained between the estimates and the actual DOA's. However, for certain satellites a considerable improvement is evidenced when compensation is used. It is important also to notice the possible presence of uncontrolled experimental conditions, like some level of multipath or other kind of signal distortions.

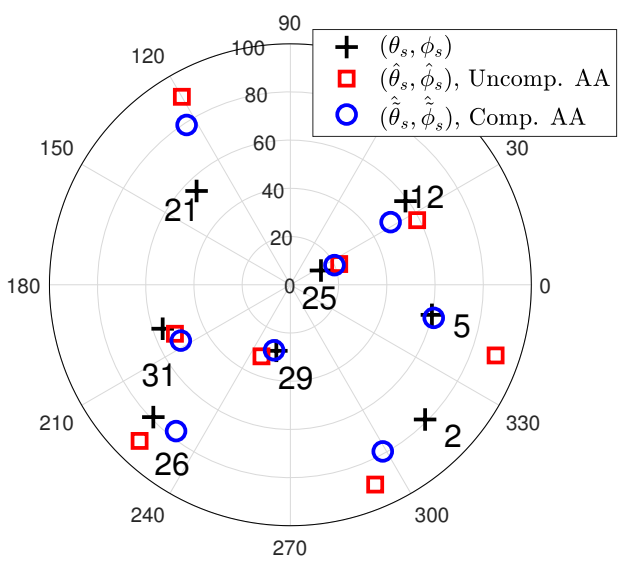

Fig. 13. True DOA's of the satellites and their estimations.

Another way to show the action of the proposed compensation is calculating the signal strength at each element of the AA. The received $C / N_{0}$ of the satellites when no transformation is applied are shown in Fig. 14.a. For each satellite, the signal strength differs considerably between the antennas, which is pretty much consistent with the simulated array response and the DOA's of the incoming signals. These differences are significantly reduced after applying the unitary transformation, as can be seen in Fig. 14.b which is also in accordance with the expected behavior.

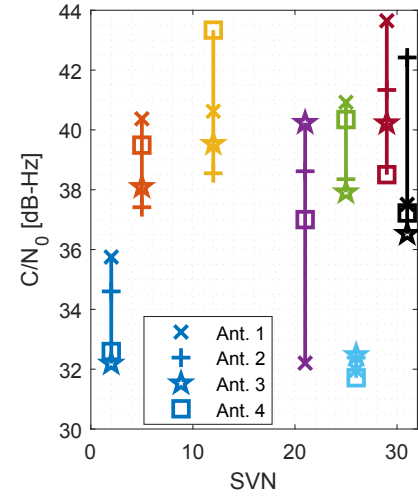

(a) Uncompensated AA

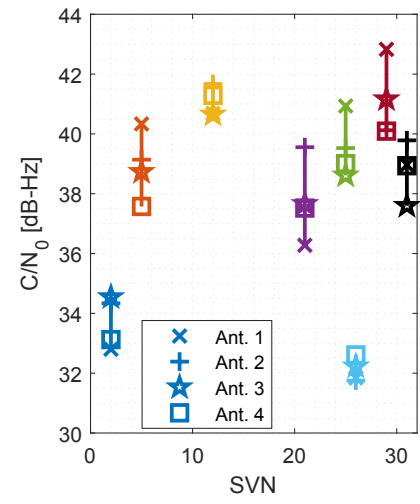

(b) Compensated AA
Fig. 14. $C / N_{0}$ of available satellites at each element of the AA.

In order to assess the AA ability of enduring adversities, we formulate an unfavorable and demanding scenario where the array must overcome the presence of a fictitious undesired source represented by one of the actual GPS satellites. The AA must be able to mitigate it at the same time that steers its main beam towards the DOA of the desired GPS signal. Therefore, the signal obtained after nullforming should have an attenuated version of the unwanted satellite signal, i.e. its power should be much lower than the other satellites. This test does not provide a measure of rejection depth, as the simulations did, though corroborates the accomplishment of the expected aim, and can be customized for spoofing, interference or jamming mitigation situations. According to the simulations, a reasonable interference rejection ratio when performing nullsteering is expected to be of about $20 \mathrm{~dB}$. Then, considering that the threshold of our detection algorithms is approximately $30 \mathrm{~dB}-\mathrm{Hz}$ of $C / N_{0}$ [34], if this level of attenuation is actually achieved the undesired signal should be undetectable. On the contrary, if the undesired signal can be detected, then the attenuation provided by the nullforming strategy will be clearly insufficient, since there are no satellites with $C / N_{0}$ as high as $50 \mathrm{~dB}-\mathrm{Hz}$.

The first test consists in suppressing the SVN's 2 and 26, while beamsteering towards each of the remaining available satellites. The choice of the two sources of interference is given by their location at low elevation angles $\left(80^{\circ}\right.$ approximately), which is more likely for a jammer or spoofer device. This process is performed by means of the OPB method, which makes use of the previously obtained DOA estimations. In Fig. 15, it is seen that the undesired sources are effectively mitigated with both schemes, while all the desired satellites have been recovered. It is clear that the relatively low signal strength of the unwanted signals makes them easy targets to mitigate, so both methods accomplish this objective.

In the following case, the SVN 12 is considered as an interference since it is located at a low elevation angle but 


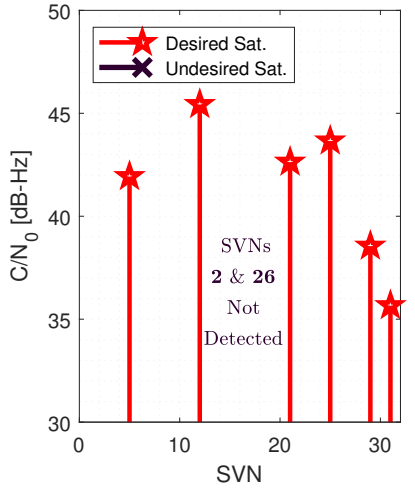

(a) Uncompensated AA

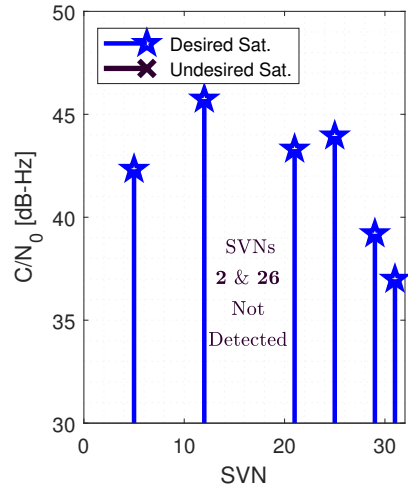

(b) Compensated AA
Fig. 15. $C / N_{0}$ of available satellites while nullsteering to SVN's $2 \& 26$.

its signal strength is relatively high. The results are presented in Fig. 16. If no compensation is applied, only two over seven satellite signals are detected while at the same time the interference (SVN 12) is low enough not to be detected. However, when the other five satellite signals are selected, the presence of the unwanted signal can still be detected. This clearly indicates that the uncompensated array cannot get rid of the interference, even that the misalignment of its DOA estimate is similar to the one of the compensated array. On the other hand, the compensated AA has detected all the available GPS signals with a slightly higher $C / N_{0}$ level and without any presence of the interferece.

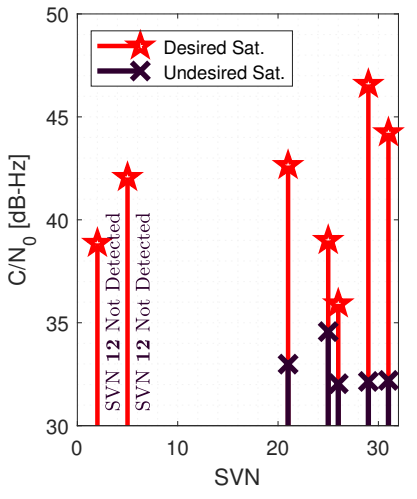

(a) Uncompensated AA

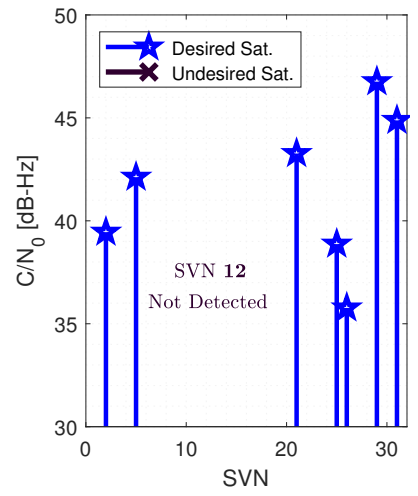

(b) Compensated AA
Fig. 16. $C / N_{0}$ of available satellites while nullsteering to SVN 12 .

As final -and most demanding- case, the SVN's 12, 21 and 31 , which are located at medium elevation angles $\left(60^{\circ}\right.$ approximately), are considered to be interferences. In Fig. 17, the differences between the performance of the compensated and uncompensated array are exhibited. Without compensation, the undesired signals were always detected showing the poor attenuation achieved. Conversely, when the compensation was applied, the undesired signals became undetectable while the $C / N_{0}$ levels of the desired signals are similar in some cases and significantly higher in others.

\section{CONCLusions}

A compact, low-cost and lightweight AA for GNSS effective spatial signal processing was presented. Given the

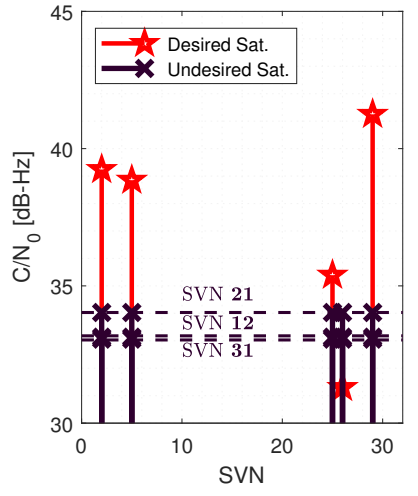

(a) Uncompensated AA

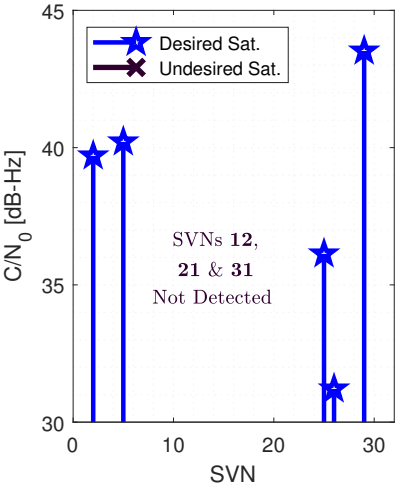

(b) Compensated AA
Fig. 17. $C / N_{0}$ of available satellites while nullsteering to SVN's $12,21 \&$ 31.

compactness of the array, the actual RP's are mostly different. Hence, the proposed AA is complemented with a transformation matrix that turns the AA into a new "virtual" array with almost identically behaved elements. Moreover, since this transformation is unitary, it does not modify the statistical properties of the received noise. The equivalent virtual array relaxes the need of employing the actual RP's information in order to allow simple array signal processing while keeping a good performance of beamforming and DOA estimation techniques with a compact AA. The proposed AA was built and validated with the experimental tests. Moreover, these tests were done using the compensation parameters that were obtained with the simulated AA response, which can be seen as an indication that the performance of the compensated array is not much sensitive to mismatch errors.

It is remarkable that the differences between the actual RP's -that are induced by the electromagnetic coupling- are in fact translated into a larger uncoupled AA. And therefore, this implies that the proposed AA has higher spatial resolution than it would be expected by its geometry. This fact can have deep consequences because it seems to indicate that certain uncoupling strategies can actually be suboptimal.

Finally, it is important to remark that the proposed approach is not restricted to GNSS applications, neither to microstrip antennas. Similar virtues on other compact AA designs can be achieved and exploited in countless applications following a similar procedure. Although our current proposal consist of using a single transformation for global compensation, a different trade-off between computational complexity and precision achieved with the virtual model can be easily chosen instead. Either adding more tuning parameters for the virtual array, or performing sectorial and time variant compensations, are valid options to obtain more accurate virtual models, that will be pursued elsewhere.

\section{ACKNOWLEDGMENTS}

The authors would like to thank Juan G. Díaz for his help with the data acquisition platform, Javier A. Smidt for his GPS signal acquisition algorithm, and the support of the rest of the SENyT members. We also express our gratitude to 
the associate editor and reviewers whose valuable comments helped us to improve the quality and clarity of this paper.

\section{REFERENCES}

[1] E. Kaplan and C. J. Hegarty, Understanding GPS. Principles and applications, 2nd ed. Boston - London: Artech House, 2006.

[2] F. Dovis, GNSS Interference, Threats and Countermeasures. Norwood, MA: Artech House, 2015.

[3] G. X. Gao, M. Sgammini, M. Lu, and N. Kubo, "Protecting GNSS Receivers from Jamming and Interference," Proc. IEEE, vol. 104, no. 6, pp. 1327-1338, Mar. 2016.

[4] J. Arribas, C. Fernandez-Prades, and P. Closas, "Antenna array based GNSS signal acquisition for interference mitigation," IEEE Trans. Aerosp. Electron. Syst., vol. 49, no. 1, pp. 223-243, Jan. 2013.

[5] M. Amin and W. Sun, "A Novel Interference Suppression Scheme for Global Navigation Satellite Systems Using Antenna Array," IEEE J. Sel. Areas Commun., vol. 23, no. 5, pp. 999-1012, May 2005.

[6] S. Daneshmand, "GNSS Interference Mitigation Using Antenna Array Processing," Ph.D. dissertation, Calgary, 2013.

[7] J. Zhang, X. Cui, H. Xu, and M. Lu, "A two-stage interference suppression scheme based on antenna array for GNSS jamming and spoofing," Sensors (Basel), vol. 19, no. 18, Sep. 2019.

[8] A. Konovaltsev, D. S. De Lorenzo, A. Hornbostel, and P. Enge, "Mitigation of continuous and pulsed radio interference with GNSS antenna arrays," in 21 st Int. Tech. Meet. Satell. Div. Inst. Navig. ION GNSS 2008, pp. $1291-1300$

[9] J. L. Volakis, A. J. O'Brien, and C. C. Chen, "Small and Adaptive Antennas and Arrays for GNSS Applications," Proc. IEEE, vol. 104, no. 6, pp. 1221-1232, Jun. 2016

[10] S. Irteza, M. Ibraheam, T. Harz, Y. Bulbin, R. Stephan, and M. Hein, "Compact Satellite Navigation Antenna Array using Off-the-Shelf Ceramic Patch Antennas," in 9th Eur. Conf. Antennas Propag., Lisbon, 2015.

[11] K. L. Chung, Y. Li, and C. Zhang, "Broadband artistic antenna array composed of circularly-polarized Wang-shaped patch elements," AEU Int. J. Electron. Commun., vol. 74, pp. 116-122, Apr. 2017.

[12] S. Caizzone, G. Buchner, M. S. Circiu, M. Cuntz, W. Elmarissi, and M. E. Pérez, "A Miniaturized Multiband Antenna Array for Robust Navigation in Aerial Applications," Sensors (Basel), vol. 19, no. 10, May 2019.

[13] I. J. Gupta, I. M. Weiss, and A. W. Morrison, "Desired Features of Adaptive Antenna Arrays for GNSS Receivers," Proc. IEEE, vol. 104, no. 6, pp. 1195-1206, Jun. 2016.

[14] E. A. Marranghelli, G. Ramon Lopez La Valle, and P. A. Roncagliolo, "A Spatial Signal Processing Review for Practical GNSS Antenna Arrays," in 2018 IEEE Bienn. Congr. Argentina, ARGENCON 2018, San Miguel de Tucumán, Argentina.

[15] H. L. Van Trees, Optimum Array Processing - Part IV of Detection, Estimation and Modulation Theory. New York, NY: Wiley, 2002.

[16] T. Sarkar, M. Wicks, and R. Bonneau, Smart Antennas. New Jersey: Wiley, 2005

[17] A. J. Weiss and B. Friedlander, "Performance Analysis of Spatial Smoothing with Interpolated Arrays," IEEE Trans. Signal Process., vol. 41, no. 5, pp. 1881-1892, May 1993.

[18] E. A. Marranghelli, G. R. López La Valle, and P. A. Roncagliolo, "Orthonormal method for compact Global Navigation Satellite Systems antenna array designs," Int. J. RF Microw. Comput. Eng., vol. 29, no. 12 , Dec. 2019.

[19] X. Tang, K. Mouthaan, and J. Coetzee, "Tunable decoupling and matching network for diversity enhancement of closely spaced antennas," IEEE Antennas Wirel. Propag. Lett., vol. 11, pp. 268-271, Feb. 2012.

[20] C. H. Wu, C. L. Chiu, and T. G. Ma, "Very Compact Fully Lumped Decoupling Network for a Coupled Two-Element Array," IEEE Antennas Wirel. Propag. Lett., vol. 15, pp. 158-161, May 2015.

[21] M. Ibraheam, B. Bieske, K. Blau, E. Schafer, A. Jager, S. I. Butt, R. Stephan, and M. A. Hein, "Feasibility of dual-polarized antenna arrays for GNSS receivers at low elevations," in 11th Eur. Conf. Antennas Propagation, EUCAP 2017, Paris, pp. 857-861.

[22] D. D. F. Kelley and W. L. W. Stutzman, "Array Antenna Pattern Modeling Methods That Include Mutual Coupling Effects," IEEE Trans. Antennas Propag., vol. 41, no. 12, pp. 1625-1632, Dec. 1993.

[23] R. Garg, P. Bhartia, I. Bahl, and I. Apisak, Microstrip Antenna Design Handbook. Norwood, MA: Artech House, 2001.

[24] Rogers Corporation, "RO4000 ® Series High Frequency Circuit Materials Datasheet," Chandler, Arizona, USA.
[25] D. G. Luenberger and Y. Ye, Linear and Nonlinear Programming, 3rd ed. New York, NY: Springer, 2008

[26] B. Friedlander, "Direction finding using an interpolated array," Proc. IEEE Int. Conf. Acoust. Speech Signal Process. ICASSP, vol. 5, pp. 2951-2954, May 1990.

[27] M. Yang, X. Zhu, Y. Zhang, N. Ta, and Z. Rao, "Parameter study of timedelay magnification in a biologically inspired, mechanically coupled acoustic sensor array," J. Acoust. Soc. Am., vol. 140, no. 5, Nov. 2016.

[28] A. R. Thompson, M. J. M., and G. W. Swenson, Interferometry and Synthesis in Radio Astronomy, 3rd ed. Cham: Springer, 2017.

[29] E. A. Marranghelli, G. R. L. La Valle, and P. A. Roncagliolo, "Mutual coupling and electromagnetic diffraction influence on GPS microstrip antenna arrays," in 16th Work. Inf. Process. Control. RPIC 2015, Cordoba.

[30] R. Schmidt, "Multiple Emitter Location and Signal Parameter," IEEE Trans. Antennas Propag., vol. 34, no. 3, pp. 276-280, Mar. 1986.

[31] A. Konovaltsev, S. Caizzone, M. Cuntz, and M. Meurer, "Autonomous Spoofing Detection and Mitigation with a Miniaturized Adaptive Antenna Array," in Proc. 27th Int. Tech. Meet. Satell. Div. Inst. Navig. ION GNSS 2014, vol. 4, Florida, Sep. 2014, pp. 2937-2948.

[32] J. G. Díaz, G. R. López La Valle, S. Rodríguez, G. Scillone, L. Mártire, E. M. López, G. L. Puga, J. Cogo, J. A. Smidt, J. G. García, and P. A. Roncagliolo, "An Experimental High Precision GNSS Receiver for Small Satellites Navigation," in 1st IAA Lat. Am. Symp. Small Satell., Buenos Aires, 2017.

[33] J. G. Díaz, G. Scillone, and J. G. García, "Multi-channel FPGA acquisition system for SDR receivers," in Argentine Conf. Autom. Control, Buenos Aires, 2018.

[34] J. Smidt, S. Ozafrain, P. A. Roncagliolo, and C. Muravchik, "New Technique for Weak GNSS Signal Acquisition," IEEE Lat. Am. Trans., vol. 12, no. 5, pp. 889-894, Aug. 2014.

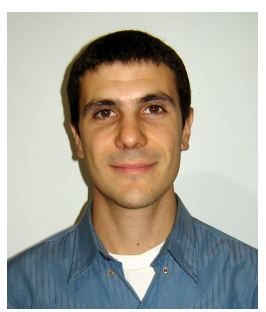

Marranghelli Ezequiel Alejandro received the Electronics Engineering degree from the National University of La Plata (UNLP), Argentina, in 2012. $\mathrm{He}$ is currently an Assistant Professor in the UNLP. His current research interests include smart antenna arrays for Global Navigation Satellite Systems (GNSS), electromagnetic antenna mutual coupling and array signal pre-processing.

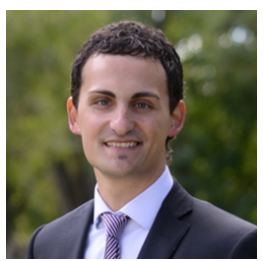

Gerardo Ramón López La Valle received the Electronics Engineering and the M.Sc. in Engineering degrees from the National University of La Plata (UNLP), Argentina, in 2008 and 2014. He is an Assistant Professor in the UNLP involved in research and development of Global Navigation Satellite Systems (GNSS) receivers for aerospace applications. His research interests are in electromagnetic compatibility, antennas, and RF and microwave circuits with applications to GNSS and wireless communications.

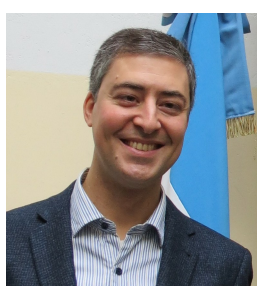

Pedro Agustín Roncagliolo received the Electronics Engineering and the Ph.D. in Engineering degrees from the National University of La Plata (UNLP), Argentina, in 2001 and 2009. Currently, he is the Associate Dean of the Engineering School at the UNLP and Head of Group Sistemas Electrónicos de Navegación y Telecomunicaciones (SENyT) involved in research and development of Global Navigation Satellite Systems (GNSS) receivers for aerospace applications. His research interests are in Statistical Signal Processing and Radio Frequency Electronics with applications to Wireless Communications and GNSS. 